\title{
Le corps du personnage
}

\author{
Nicole Brossard
}

\section{The body/core of the character}

The body is our solitude, writes Nicole Brossard, our only certitude, but because the body has eyes for seeing, ears for hearing, a memory for storytelling, and words for drawing relationships, we are not alone... The body is gregarious, but it insists upon its singularity, its intimacy. Take that away and you take away its honour. The body likes to be seen, but reduce a person to her/his body and you humiliate the person.

In this text, Brossard remembers the body at various moments in her writing: the so-called neutral body of modernity that had no gender other than that of sensuality, transgression, and subversion; the body of the mother, heavy with the weight of identifying as a woman; the lesbian body, an aerian body that displaces itself constantly in the direction of another woman; and the body of writing, a body trying to escape the body through language.

Dear, dear! How queer everything is to-day! And yesterday things went on just as usual. I wonder if I've changed in the night? Let me think: was I the same when I got up this morning? I almost think I can remember feeling a little different. But if I'm not the same, the next question is, Who in the world am I? Ah, that's the great puzzle!

- Lewis Carroll, Alice in Wonderland

Entre Le Désert mauve (1987) et Baroque d'aube (1995), mon corps aura vieilli de sept ans. Suis-je la même femme, la même poète, romancière? Combien de personnages faut-il pour transformer un corps et les traits du visage? Combien de naissances pour transformer une femme? Combien d'orgasmes pour transformer un «je»? Combien de deuils pour faire entrer la vie comme un principe de plus dans la pensée du récit? Combien de voyages à la mer pour imaginer le silence?

Le corps est notre solitude, notre seule certitude. À partir de son bienêtre, de son autonomie, de son mal-être, nous faisons notre philosophie 
de vie. Mais puisque le corps a des yeux pour voir, des oreilles pour entendre, une mémoire pour fabuler et des mots pour comparer, voilà que déjà, nous ne sommes plus seuls et que, dans l'agitation ambiante, le corps apprendra, pour le meilleur et pour le pire, à se frotter à autrui.

Entre le corps-tombeau de Platon, le corps-théâtre, le corps d'écriture de la modernité, le corps féminin de différence, le corps d'utopie lesbienne, le corps de spectacle queer et le corps de performance rêvée, le corps invente sa survie, son récit, c'est-à-dire son déplacement au milieu du savoir et des croyances. Il faut placer le corps au bon endroit dans la part de rêve qui nous habite.

Dès les premiers jours, commence un récit dont nous connaissons pourtant le dénouement. Le récit le repousse en faisant des retouches à l'enfance, à l'amour, à la nature. La poésie nous réinvente jusqu'à l'os et la poussière d'étoiles. C'est toujours une histoire du corps que nous racontons: corps de libido, corps de souffrance, corps de tendresse, corps de tempête, corps perdu, corps retrouvé comme une origine.

Nous n'avons qu'un seul corps, pourtant, il y a trop de corps en nous, dirions-nous, pour que nous n'en détournions pas quelques-uns à notre avantage en les enrobant du verbe être, espérant qu'ils puissent ainsi s'épanouir dans les mille et une versions imaginées de l'identité, de la beauté et de notre goût indiscutable pour l'éternité.

Le corps est ce qui reconnaît le mieux au toucher notre vraie nature. Tout le reste ça se discute, ça se négocie par fantasmes interposés, puisque dans une très large mesure le corps travaille sans répit dans la luxuriance de l'imaginaire, le chi-chi des trames idéologiques et les révélations de la science toute-puissante.

Le corps, il est influençable. Il y a tant de mots pour lui faire croire qu'il peut, qu'il doit faire ceci ou cela, qu'il doit endurer ça. Tant de mots pour augmenter ou diminuer son seuil de tolérance. Oui, il est grégaire le corps. Des gestes aussi simples que marcher, manger, d'autres, plus intimes comme pleurer, rire, séduire sont fort teintés de culture. Nous faisons tous la même chose en pensant que nous sommes différents. Nous sommes plusieurs à nous croire unique et cela crée tellement d'ambiguïté que nous glissons imperceptiblement du «je» au «nous», de «nous autres» à «vous autres» en parlant de liberté et de futur. Il est grégaire le corps, mais il tient à sa singularité, à son intimité. Lui enlever cela, c'est le déshonorer. Il aime se faire voir le corps, mais réduire quelqu'un à son corps, $c^{\prime}$ est l'humilier.

Le corps, il a du poids, parfois même un poids moral. Un ultime argument de protestation: circuler nu dans un endroit public, s'immoler par 
le feu sur la place publique, faire la grève de la faim. Le corps ne parle jamais pour ne rien dire. $S^{\prime}$ il parle tout bas, on peut faire semblant de l'ignorer mais s'il crie, il est terrifiant. Le pire, c'est quand il écrit et qu'il se fait dire qu' une image vaut mille mots.

Il a quand même fait un étrange bout de chemin le corps de l'espèce! Tout cela en s'inventant de petits et grands conforts: un petit bras en bois pour se gratter le dos, des bras d'esclaves ou un sexe féminin en forme de main d'oeuvre et toute une série d'objets: outils et jouets comme la roue, le télescope, le microscope, qui en prolongeant les possibilités du corps, l'aide à mieux comprendre sa petitesse et sa fragilité sans pour autant nous inciter à l'humilité.

Mais avant tout, le corps, il aime bien se voir. Miroir, une plume et une feuille, une caméra, il y aura toujours quelqu'un pour se pencher sur la question de la représentation. Ça ne suffit pas? $D^{\prime}$ accord un écran pour te mettre la puce à l'oreille et te faire oublier ta lenteur naturelle. Quoi encore? Je voudrais des sensations fortes. Voici quelques adresses, un Joy Stick et un Hand Master. Oui mais ici, il manque un rein, un coeur, des yeux. Ma foi, les voici pour un bon prix. Il manque un enfant, voilà maman porteuse, elle aussi pour un bon prix. Il manque quoi encore?/ Rien. J'ai peur de mourir./ T'exagères. Excuse-moi, j'ai un rendez-vous. But don't worry we all die the dark side in us shattered by love.

Combien de corps ai-je connus dans ma vie d'écriture?

\section{Corps d'écriture f'eros}

J'ai souvent dit que pour moi l'aventure de l'écriture avait commencé avec mon troisième recueil de poèmes L'écho bouge beau (1968). Or, dans ce recueil, j'affirmais comme une déclaration de principe: «Neutre le monde m'enveloppe neutre». Étrange affirmation pour une poète valorisant la sensualité et intéressée par le politique. Qu'ai-je donc voulu dire? Sans doute que je donnais préséance à l'écriture pour m'inventer, c'est-à-dire que j'étais prête à reléguer au second plan tout ce qui constitue l'anecdote du vivre et du biographique pour faire place à l'écriture, à la passion des mots, à leur fétichisme.

En fait, à partir du moment où se dessine dans mes textes, l'ambition du neutre (somme toute, une bonne formule pour refouler le féminin), le mot corps deviendra mot-fétiche dont la tâche sera de revendiquer pour le corps sexuel une place de choix sur la place publique. Le corps sexuel tenant lieu, dans la société encore puritaine du Québec des années soixante, de symbole libertaire. Bien évidemment, je comprendrai plus 
tard que la prétention au neutre ne fait taire qu'un seul genre: le féminin.

C'est ainsi que de 1968 à 1974, le corps dit neutre de l'auteur, porté comme un flambeau de modernité, $n$ 'aura de genre que celui de la sensualité, de la transgression et de la subversion tenues comme moyens privilégiés pour assurer au corps sexuel toute la place qui lui revient en société. Faire advenir du sexuel à partir de l'écriture jusqu'à ce que le sexuel et l'écriture deviennent dans un chassé-croisé d'arguments ludiques, une plaque tournante de la politique du plaisir comme source de lucidité.

En somme, ce premier corps d'écriture il sera tout à la fois textuel, ludique, provocateur et iconoclaste. Il n'est que de songer à quelquesuns de mes titres de l'époque: La verge au beau tarif, Vaseline, Calligraphe/travesti. Et je ne suis pas sans remarquer que ce sont des titres étonnamment «gais» pour une jeune femme poète.

Bien sûr, je simplifie, car dans mes premiers textes, le mot corps aura toujours valeur d'énergie. Tantôt il signifiera ardeur concentrée, tantôt il faudra l'associer à la fébrilité ralentie par le pur bonheur de l'instant, tantôt on pourra y voir une intention d'harmonie avec le monde comme dans Suite logique et Le Centre blanc (1970). Ce premier corps d'écriture, il sera tenté par l'excès, $l^{\prime}$ absolu, habité par l'obsession du présent comme seule garantie d'intensité, de plaisir et de lucidité. En fait, mon corps n'aura de genre que celui d'un «je» profitant de la vie avec un grand appétit et cherchant dans la langue les sensations fortes que ne manque jamais de procurer la magie des mots. Surtout si on les convoite côté signifiant.

\section{Le corps de la mère}

Écrire, je suis une femme, est plein de conséquences. - L'amèr ou le chapitre effrité (1977)

Cette fois-ci, le corps parle en direct. Le neutre a disparu, le féminin déborde. Le corps n'est plus une abstraction. Il fait désormais poids de réalité. Il y a du plein partout. Du plein temps, du plein de matière quotidienne. La mère que je suis est maintenant un autre corps, au milieu d'un grand tout qui la réinsère dans le grand ensemble des femmes, pour ne pas dire des personnes de son sexe. Je vois bien soudain que donner la vie c'est engendrer la question du genre et que cela ne va pas sans vertige et tremblement du sens.

En devenant mère, c'est tout le poids de l'humanité des femmes qui 
m'est tombédessus. Tout le poids du destin de la femelle. Toute la pesanteur patriarcale, les énormes gros sabots de tradition et de culture piétinant la vie et l'énergie des femmes. Poids de réalité. Et oui, ça m'est venu soudain l'idée que moi aussi j'étais une femme, que j'étais comme des millions des femmes, un corps marqué, marginalisé et ciblé.

Voilà que soudain le féminin règne comme une ambiance. La différence éclate au grand jour. Le corps au féminin est désormais incontournable. Je suis au coeur du sujet et de la nature; plus est, je suis au coeur du symbolique, question de vie, question de mort. C'est ici que ça se complique. Car, nous sommes dans le mensonge. Rien ne va plus. Il y a des imposteurs. L'imposture est dans la langue, le symbolique et l'imaginaire. Comment trouver les mots pour dire: je $\mathrm{m}^{\prime} \mathrm{en}$ aperçois. Comment départager la fiction de la réalité? Comment faire sens comme sujet féminin dans une langue qui me rend invisible et qui simultanément me pointe du doigt.

La tension sémantique est à son comble. Il faudra faire travailler la langue et non plus travailler le texte-fétiche.

Il faudra s'attaquer à la représentation. Soulever une à une les couches sémantiques de malheur qui recouvrent les femmes. Couper dans le gras du phallocentrisme. Il faudra y mettre du mien au risque de ne pas $m^{\prime}$ en remettre, de passer pour encore plus folle et dangereuse que sorcières, furies, mégères et mille autres formes du féminin désignées maléfiques au nom du père et du fils.

Il faudra prendre des risques. Cette fois-ci non pas pour le «kick» du risque mais pour comprendre d'où origine le mépris des femmes et comment il s'est installé avec autant d'aisance dans le quotidien culturel.

\section{Le corps lesbien}

excitation: ce qui me suscite inédite dans ma peau.

$$
\text { - Amantes (1980) }
$$

je pensais réellement comme un peau qui vit sa raison d'être. - Picture Theory (1982)

Tout corps porte en lui un projet de haute technologie sensorielle qui est son hologramme.

- La Lettre aérienne (1986)

Une fois commencé le travail de présence à l'autre femme, le corps a des ailes pour la rejoindre, des bras, des genoux, des cuisses pour la retenir, une peau si lisse que c'est comme une marque personnelle de confiance 
en chaque femme. Une fois que le désir s'en mêle, toute l'âme y passe et le corps acquiert une incroyable légèreté, parfois même une certaine transparence. Le corps devient aérien. Voyageur, il peut passer des jours entiers à rêver d'utopie. Toujours ailleurs. Là où le chant de l'amante le porte. C'est ardeur concentrée et certitude, emportement et ravissement. Bref, la vie à un très haut niveau de conscience et d'exubérance. C'est la pensée de l'émotion et l'émotion de la pensée qui feront apparaître la voix, le souffle et la peau comme des petites merveilles de singularité et d'intégrité.

Le corps lesbien soulève tout sur son passage: les pensées, les images. Il naviguera dans la langue avec une adresse de sujet radieux. Tant mieux.

Corps en harmonie.

Là où il y a un corps heureux, elle peut s'abandonner dans l'abondance des abstractions, et s'en donner à coeur joie de toutes les audaces et débordements de sens. Je n'y peux rien. J'associe le corps lesbien à un corps d'intelligence radicalement porté sur la chose de l'utopie et de l'espoir. Je n'y peux rien, et à la vérité j'aime bien répéter que c'est mon corps lesbien qui m’a donné les meilleures idées, inspiré les plus beaux délires, transformé le regard en vision.

Soumis à la loi de la gravité avec son ventre, ses seins et son sexe de reproduction, le corps de la mère est absolument conforme à des milliers d'autres corps féminins, alors que le corps lesbien sera aérien, ravi que chaque femme soit si singulière et omniprésente, familière et étrangère. Là où le corps de la mère est un féminin pluriel qui nourrit les pensées profondément morales, responsables et solidaires de la féministe, le corps lesbien alimente des fantaisies sans nom qui la rapprochent du poème, qui la situe d'emblée du côté de l'insoumission, la déplace constamment vers l'autre femme.

\section{Le corps décriture}

car les mots peuvent brûler le corps, en faire leur fièvre précieuse pour toujours un peu plus s'avancer dans les territoires de feu, jusqu'à frôler l'irréel. (1979)

L'écriture c'est du corps. Et ce à plusieurs niveaux. Le premier, très physique.

C'est bien connu, plusieurs sont littéralement malades, maux de ventre, nausées, vertige lorsqu'elles ou ils écrivent. D'autres entrent 
dans un tel état d'excitation sexuelle que la sève monte. Il y a aussi les larmes quelquefois. Sans doute aussi des bras levés en signe de victoire quand un mot est on ne peut mieux choisi. Il y a l'adrénaline, la concentration, le corps en état second, une conscience altérée du temps.

Il y a aussi ce qu'on appelle faire passer du corps dans la langue, et ce n'est pas rien. Le pire c'est qu' on ne parvient pas vraiment à tricher. On aura beau faire mille tours de passe passe littéraire, le corps dicte au présent s'il est heureux, au passé s'il en a trop sur la mémoire. Au futur, on ne sait jamais où il veut en venir exactement. C'est d'ailleurs parce qu'il est impossible de tricher que nous recommençons, ressortons les mêmes cartes. Dans l'écriture, nous reprenons le corps essentiel pour qu'il fasse cause commune avec le corps inquiet et le corps glorieux des sens.

Au risque de me contredire, je dirais que l'écriture c'est du corps qui tente d'échapper au corps par le langage, du corps qui cherche à traduire le "c'est tellement, tellement» et tout autre formule tautologique du genre «la douleur, c'est la douleur, une femme est une femme, etc.»

J'avais une amie qui, sachant que j'étais en amour, me disait d'un air moqueur: «N'oublie pas: un orgasme c'est une page d'écriture en moins». Au fond, elle reprenait le cliché par lequel on disait quel'homme payait de son génie le temps qu'il consacrait à l'amour, c'est-à-dire à la femme. Bien sûr, elle avait tort, car qui écrit trouvera tôt ou tard, les mots pour sortir de la tautologie, ne restera pas bouche bée de bonheur ou immobilisé dans le cri rauque de la souffrance. Écrire, c'est dénouer, ouvrir. C'est faire passer.

Tout ce que le corps a connu en direct, c'est-à-dire par les sens, est inscrit à tout jamais dans la mémoire. Tatouage de sensations et d'émotions. Images qui surgissent à l'improviste. C'est tout ça qui se retrouve transformé, "processé» dans la langue de manière à ce que tout le monde puisse en profiter.

Dire «je voudrais» fait travailler le corps. En somme, le désir.

$\mathrm{Au}$ fond, il y a quelque chose de très beau à imaginer que nous pouvons délirer avec exactitude. Il y a quelque chose de troublant à oublier notre corps pour mieux laisser vivre le personnage. Quoique faire entrer du personnage dans nos vies, épuise aussi. Il faut alors comprendre ce qui nous unit aux êtres chers imaginés avec fureur et délice comme si on allait perdre son identité, gagner en humanité, augmenter le poids charnel de l'univers.

Impossible de laisser le personnage tranquille. Son inquiétude doit être exemplaire. Il faut lui tailler un berceau et une tombe à sa mesure. En 
attendant, lui trouver un appartement, arroser ses violettes au balcon de manière à ce que lectrices et lecteurs remarquent le bel ensemble. Il faut l'habiller, passer sa vie au peigne fin, la sortir du pétrin, la regarder creuser son avenir au milieu de la violence et des villes illuminées. Il arrive parfois que le personnage accouche $d^{\prime}$ un autre personnage qui nous prend au dépourvu tant et si bien qu'on songe à laisser faire la réalité, parfois même sa propre histoire, son propre corps.

Devant la fenêtre, dénouant ses cheveux, ouvrant son courrier, un tiroir ou le fond de sa pensée, le personnage n'en finit plus d'exister. Objet d'enquête, elle se laisse toucher plus facilement à partir de l'enfance parce qu'elle commence toujours à peu près là à grandir.

D'accord. Mais que peut le personnage alors que nous franchissons quotidiennement des seuils imaginaires qui éloignent la mort et la rapproche, gros plan à l'écran, plus présente que jamais. Que peut le personnage après avoir imaginé les corps mystérieux de la sirène, du minotaure, du centaure, de l'ange et du démon, du vampire et de l'androgyne? Que diront les poètes en s'apercevant que l'ardeur du soleil sur leur peau se calcule en uva, que la mer est plus que jamais en allée sous son manteau de pétrole? Que feront les yeux quand ils ne sauront plus distinguer le vrai du faux, entre l'artifice et l'artifice? Bien sûr, il faudra réapprendre. Je veux bien, c'est la moindre des choses quand on est vivant.

L'obsession contemporaine du corps c'est notre nez à nez avec la technologie. À force de perfectionner des petits bras pour nous gratter le dos, nous nous sommes dotés de longs bras électriques pour mieux nous brancher à l'univers. Pulsion. Cela n'est jamais dit clairement mais quelqu'un rêve quelque part de naître sans nombril.

L'écart entre soi et le monde diminue. Le désir recule devant les avantages de l'ici et maintenant de la sensation. Il faut se pincer de plus en plus fort pour être bien sûr qu'on est dans le vrai.

Pourtant, nous avons atteint notre grandeur nature. Comme le dit le philosophe Michel Serres: «Nous voici parvenus à des tailles telles que nous existons enfin physiquement». À la nature, nous répondons de plus en plus concrètement. Nous vivons dans l'excédant du corps et nous nous terrons dans le corps quand trop d'abstraction nous affole. Cela dit, il reste peut-être l' ego pour faire corps avec le corps. Alors, en terminant, elle dira:

Je suis une femme du présent, côté ludique d'où il est possible d'apercevoir entre les mots, nos petites superstitions, notre belle morale, 
nos grands dada en pleine action. Je suis une urbaine, côté graffiti du mur, côté nuit blanche, côté paroles en liberté. Côté corps où le monde tangible se révèle sous des angles inconnus, plein de ressources: côté écriture où la peau est une fervente collectionneuse d'aubes et de rires, d'odeurs anciennes et d'idées neuves.

J'écris pour faire acte de présence dans la langue. Pour que le vivant l'emporte. Que dans l'épreuve du vivant, les mots soient fureur d'où jaillissent mille présences suggestives qui tombent bien au milieu des pensées.

J'écris parce que c'est dans la langue qu'il faut deviner, gagner notre humanité. J'écris parce que le présent est incommensurable, habité en permanence d'aubes et de nuits, de siècles et de cultures. Précieux présent qui garde les sens en alerte, présent comme un récit au milieu des analogies, présent qui fait son miroir, présent qui traque en nous le personnage.

Alors, elle conclura: Un seul corps pour nous instruire du plaisir. Un seul corps pour la présence et l'absence, pour découper dans le temps la forme accidentées des pensées. Un seul corps pour satisfaire l'envie de lumière et de mer. Un seul corps pour trouver les mots nécessaires, pour nous obliger à répéter. Le même pour comparer. Corps de mémoire pour inventer et progresser vers le silence. Dans quelques instants, j'existe encore.

Or, tous les jours, le corps se transforme à notre insu. Les pensées changent-elles de nature parce que le corps attrape à d'autres niveaux le sens de la vie? Le corps peut-il simultanément faire attention aux choses universelles, à la couleur de l'aube et laisser faire la fiction?

Dans quelques instants, j'existe encore. My life is all about life. Language is alive in my throat. Can you hear the vibration? My voice has been severely damaged by a dream. I used to dream among dreamers. The dreamers have left. What about you? Rêvez-vous, dit-elle, encore.

Ce texte a été présenté pour la première fois dans le cadre du Sixième colloque annuel des étudiantes et étudiants des deuxième et troisième cycles de l'Association canadienne de littérature comparée ( «Corps/Corpus. The Body of Literature/Literature of the Body"), tenu au Département de littérature comparée de l'Université de Montréal les 31 mars et 1er avril 1995 et organisé par Marie Lessard et Craig Ireland. 